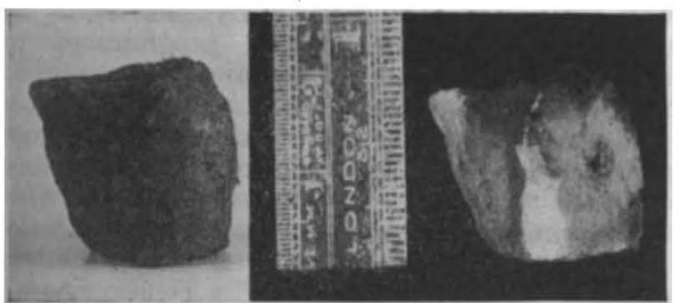

Fig. 1.

Fig. 2.

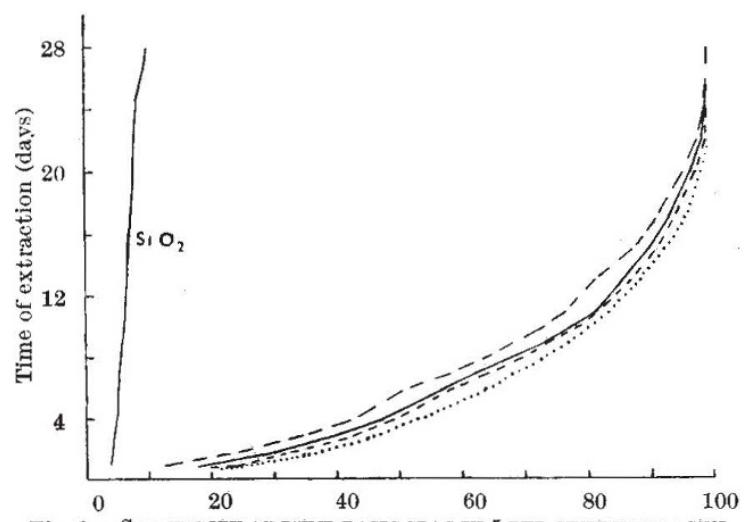

Fig. 3. SOLUBILITY OF LUMP BASIC SLAG IN 5 PER OENT HYDROGEN CHLORIDE SOLUTION.

$\mathrm{Fe}, \longrightarrow ; \mathrm{CaO}, \ldots-\cdots ; \mathrm{MnO}, \ldots \ldots ; \mathrm{P}_{2} \mathrm{O}_{5},-\longrightarrow-$

silica, 45.51 ; ferrous oxide, $0 \cdot 90$; alumina, $23 \cdot 10$; manganese oxide, $28 \cdot 52$ per cent.

When extracted by warm, concentrated hydrochloric acid, these slags yield white, glossy residues of pure silica.

Further interesting phenomena were observed during an investigation dealing with hæmatite iron ores. Ordinary lump ore samples when extracted in concentrated hydrochloric acid, as above, leave a rigid porous skeleton of pure silica; this residue has a crystalline appearance. Samples of kidney and pencil ores from the same mine, when thus extracted, leave the silica in a laminated, transparent, gelatinous form.

The information, here available, may shed some light on this very obtuse problem and be of value to future investigators.

Steel, Peech and Tozer,

B. W. Methley.

H. J. TURNER.

The Ickles,

Sheffield, 1.

May 4.

\section{Non-Solar Planetary Systems}

IN his communication in NATURE ${ }^{1}$ on non-solar planetary systems, Sir James Jeans writes, "Mr. Sen refers with approval to Banerji's modification of this [tidal] theory. This makes the original sun a Cepheid which had its oscillatory instability increased by the gravitational attraction of a passing star. But a passing star cannot increase the instability of a Cepheid appreciably unless it comes very near. . . Judging from their luminosity, all Cepheids are much more massive than the sun or 61 Cygni or 70 Ophiuchi."

In my paper" on "The Instability of Radial Oscillations of a Variable Star and the Origin of the
Solar System", I have given a theory which, I believe, satisfactorily accounts for the amount of angular momentum and energy possessed by the planets. The sun is supposed to have been originally a part of a Cepheid variable much heavier than the sun, which oscillated radially with small amplitude. It has been shown mathematically that the oscillations are stable only if the amplitude is so small that its square may be neglected. Instability will ensue if the amplitude is increased so that its square can no longer be neglected. The nearby passage of a star of about the mass of a Cepheid increased the amplitude of the oscillations, rendering them unstable. Matter was consequently thrown out, which condensed into the sun and the planets. If the parent Cepheid had a mass about nine times that of the sun, the sun and its planets would take away about two fifths of the Cepheid's energy. It has also been shown that the encounter need not be "very close", nor need the intruding star have an inordinately large velocity to give the requisite angular momentum to the sun and the planets and enough energy to the solar system to escape from the parent Cepheid. It appears that due to the difficulties of transit at present, Sir James Jeans has not perhaps seen my paper.

Jeans has also suggested ${ }^{3}$ that the sun was originally big enough to include the orbit of Uranus and yet not too diffuse to hang together dynamically. There are several difficulties in such a theory which cannot be explained satisfactorily. The angular velocity imparted to the surface of the primitive sun extending up to the orbit of Uranus will presumably be of the same order as the angular orbital speed of Uranus. If the sun contracted from its enormous original size to its present dimensions, its angular velocity should increase considerably. Even granting that 98 per cent of the original angular momentum has been taken away by the planets, a little calculation will show that the sun should be rotating with at least 400 times its present angular velocity and should on that account become distinctly spheroidal in shape.

Moreover, with such rapid rotation, possibly the critical value for $\omega^{2} / 2 \pi \gamma \rho$ will be reached, and there will be equatorial break-up, resulting in the formation of rings of particles surrounding a central lenticular mass. Again, if the planets had taken away 98 per cent of the total angular momentum as calculated on the above theory, they should be revolving much more rapidly at present.

Another point in this theory may also be mentioned. Jeans supposes that the planets are most likely to have been formed before the sun's radius fell much below 4,000 times its present radius. It is difficult to understand what processes might have helped to bring the innermost planets to their present distances of the order of 100 times the sun's radius.

Sen has referred in his communication in NATURE ${ }^{4}$ to the well-known Jeans-Jeffreys theory, from which it follows that planetary systems should be of "the nature of freak formations"'. It seems that he did not refer to Jeans's recent communication in NATURE ${ }^{1}$, as the suggestions there are "very tentative".

Department of Mathematics,

A. C. BANERJI.

1 NATURE, 152, 721 (1943).

${ }^{2}$ Banerji, A. C., Proc. Nat. Inst. Sci. Ind., 8, 173 (1942).

${ }^{3}$ NATURE, 149, 695 (1942).

- NATURE, 152, 600 (1943).

'Jeans, J. H., "Astronomy and Cosmogony", 401 (1928). 